\title{
Schwingungsberechnungen an Cyclopropenderivaten zur Zuordnung und Charakterisierung der beobachteten Banden ${ }^{1}$
}

\author{
Zur Struktur und Reaktivität der Cyclopropenone, 5. Mitteilung \\ FrIEdRICH HÖFLER * \\ Institut für Anorganische Chemie der Technischen Hochschule Graz \\ BERNHARD SCHRADER \\ Institut für Spektrochemie und Angewandte Spektroskopie, Dortmund \\ und Adolf Krebs \\ Organisch-Chemisches Institut der Universität Heidelberg \\ (Z. Naturforsch. 24 a, 1617-1626 [1969] ; eingegangen am 14. Juli 1969)
}

\begin{abstract}
Unter Verwendung eines erweiterten Valenzkraftmodells werden Normalkoordinatenanalysen an Cyclopropenylium-Ionen $\mathrm{C}_{3} \mathrm{X}_{3}^{+}\left(\mathrm{X}=\mathrm{Cl}, \mathrm{Br}, \mathrm{C}_{6} \mathrm{H}_{5}\right)$, Tetrahalogencyclopropenen $\mathrm{C}_{3} \mathrm{X}_{4}(\mathrm{X}=\mathrm{Cl}, \mathrm{Br})$ sowie ${ }^{16} \mathrm{O}$. und ${ }^{18} \mathrm{O}$-Cyclopropenonen $\left(\mathrm{R}=\mathrm{CH}_{3}, \mathrm{C}_{6} \mathrm{H}_{5}\right)$ durchgeführt. Es werden Kraftkonstanten erhalten, die folgende Aussagen zulassen: Im Cyclopropenyliumsystem sind die Valenzkraftkonstanten der Bindungen im und am Ring größer als bei analogen Benzolderivaten. Bei den Cyclopropenonen sind die Kraftkonstanten der Doppelbindungen gegenüber den Werten in nichtkonjugierten Olefinen und Ketonen erniedrigt, diejenigen der Einfachbindungen erhöht. Daraus folgt, da $\beta$ die Betain-Struktur am Grundzustand beteiligt ist. Die Potentialenergieverteilungen und Eigenvektoren zeigen eine starke Mischung der verschiedenen inneren Koordinaten des Moleküls an.
\end{abstract}

Für die unerwartet große thermische und chemische Stabilität der Cyclopropenone wird auf Grund von Messungen der Dipolmomente und der Basizität ${ }^{2}$, der Lösungsmittelabhängigkeit der UV-Spektren $^{1}$, von NMR-spektroskopischen Ergebnissen ${ }^{2}$ und auch nach quantenmechanischen Berechnungen ${ }^{3}$ die Möglichkeit eines Bindungsausgleichs zu der „aromatischen" Cyclopropenylium-Form Ib verantwortlich gemacht.

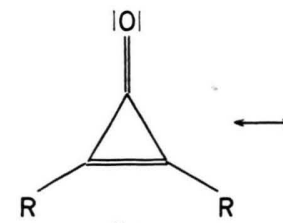

Ia

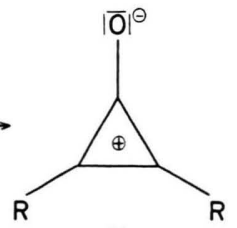

Ib
Die Infrarotspektren von Cyclopropenonen zeigen zwei sehr starke Absorptionen bei $1830-1865$ so-

Sonderdruckanforderungen erbeten an Dr. F. HöFLER, Institut für Anorganische Chemie der Technischen Hochschule Graz, A-8010 Graz/Österreich, Rechbauerstr. 12.

* 1967/68 Gast des Anorganisch-Chemischen Instituts der Universität Münster/W.

1 3. Mitt. A. Krebs, B. Schrader u. F. Höfler, Tetrahedron Letters 5935 [1968] ; 2. Mitt. A. Krebs u. B. Schrader, Liebigs Ann. Chem. 709, 46 [1967] ; 4. Mitt. A. KreBs u. J. BRECK WOLDT, Tetrahedron Letters, im Druck. wie $1590-1660 \mathrm{~cm}^{-1}$ (l. c. ${ }^{1,4}$ ). Die Bande höherer Frequenz tritt im Raman-Spektrum mit mittlerer Intensität auf und besitzt nur geringe Lösungsmittelabhängigkeit, während die tiefere Bande auch als starke Raman-Linie in Erscheinung tritt und sich deutlich nach kleineren Wellenzahlen verschiebt, wenn man von unpolaren zu polaren Lösungsmitteln übergeht. Bei der Markierung von Diphenylcyclopropenon mit ${ }^{18} \mathrm{O}$ beobachtet man bei beiden Banden eine Isotopenverschiebung (bei $1840 \mathrm{~cm}^{-1}$ um ca. 15, bei 1630 um ca. $10 \mathrm{~cm}^{-1}$ ). Daraus folgt eine sehr starke Kopplung dieser beiden die $\mathrm{C}=\mathrm{O}$ - und $\mathrm{C}=\mathrm{C}$-Koordinate betreffenden Schwingungen, die über die C-C-Einfachbindung dieses gespannten Dreiringsystems erfolgen muß ${ }^{1}$. Auf die Möglichkeit einer starken Kopplung wurde auch von anderer Seite hingewiesen ${ }^{5,6}$. Im Gegensatz dazu geben andere Zuordnungen der beiden Banden als ${ } \nu \mathrm{C}=\mathrm{O}$

2 R. Breslow u. L. J. Altman, J. Amer. Chem. Soc. 88, 504 [1966] und darin zitierte Literatur.

3 S. L. Manatt u. J. D. Roberts, J. Org. Chem. 24, 1336 [1959].

4 A. Krebs u. B. Schrader, Z. Naturforsch. 21 b, 194 [1966].

5 R. C. Lord, zitiert bei S. Andreades, J. Am. chem. Soc. 87, 3941 [1965].

6 Gerhard L. Closs, Cyclopropenes, Advances in Alicyclic Chemistry, Vol. I, 53-127, Academic Press, New York 1966. 
und $\nu \mathrm{C}=\mathrm{C}$ " $7,8,, \nu \mathrm{C}=\mathrm{C}$ und $\nu \mathrm{C}=\mathrm{O}^{\text {“ 9-12 bzw. als }}$ „charakteristische Cyclopropenonschwingung und $\nu \mathrm{C}=\mathrm{O}^{\text {“ }}{ }^{13}$ den Sachverhalt nicht richtig wieder. Am ehesten kann man nach qualitativen Betrachtungen von gleichphasig und ungleichphasig gekoppelten Schwingungen $\operatorname{der} \mathrm{C}=\mathrm{O}$ - und $\mathrm{C}=\mathrm{C}$-Bindung sprechen ${ }^{1,4}$.

In dieser Arbeit versuchen wir mittels einer Normalkoordinatenanalyse einen Einblick in die komplizierten Kopplungsverhältnisse in den Schwingungsspektren von Cyclopropenonen und einigen verwandten Molekülen zu gewinnen.

\section{Potentialfunktion}

Zur Darstellung der Potentialfunktion verwenden wir bei unseren Berechnungen ein um Wechselwirkungskonstanten zwischen den Bindungen erweitertes Valenzkraftmodell.

Um die Zahl der Variablen in der quadratischen $F$-Matrix zu begrenzen, werden alle zu Winkeländerungen gehörenden inneren Kordinaten in der Potentialfunktion vernachlässigt. In vielen Fällen führt nämlich die Kopplung der Valenzschwingung in einer Gruppe A-B - A mit der Deformationsschwingung bei einem Frequenzverhältnis $>2: 1$ dazu, daß bei der - etwa durch zusätzliche Meßdaten - bestimmten Lösung der F-Matrix der Potentialenergieanteil der Valenzkoordinate zur Valenzschwingung nahezu 1 beträgt ${ }^{14}$. Der Anteil der Deformationskoordinate an dieser Schwingung wird durch eine entsprechend negative Wechselwirkungsenergie weitgehend kompensiert. In ${ }^{14}$ wurde gezeigt, daß eine sołche Näherungsrechnung mit eliminierten Deformationsschwingungen zu prozentualen Abweichungen der Valenzkraftkonstanten von $<5 \%$ führt. Die Vernachlässigung der Winkeländerungen im Dreiring ist zugleich eine Form, die Redundanzbedingungen des cyclischen Systems zu berücksichtigen.

Zur Darstellung der kopplungsabhängigen Phenylschwingungen verwenden wir als Ersatzmodell für

7 R. Breslow, R. Haynie u. J. Mirra, J. Am. Chem. Soc. 81, 247 [1959].

8 B. E. Zaitsev, Yu. N. Sheinker u. Yu. D. Koreshiov, Ber. Akad. Wiss. UdSSR 136, 1090 [1961] ; Chem. Abstr. 55, 19480 [1961]. - B. E. Zaitsev, Yu. D. Koreshrov, M. E. Volpin u. Yu. Sheinker, ibid. 139, 1107 [1961]; Chem. Abstr. 56, 344 [1962]. - YU. G. ВоRорко u. YA. K. SYR KIN, ibid. 136, 1335 [1961]; Chem. Abstr. 55, 19743 [1961]. - YU. G. BorodKo u. YA. K. Syrkin, ibid. 134, 1127 [1960] ; Chem. Abstr. 55, 12039 [1961].

9 A. W. Krebs, Angew. Chem. 77, 10 [1965]. eine Phenylgruppe eine gewinkelte Dreimassengruppe (Abb. 1), für die wir aus Rechnungen an monosubstituierten Benzolderivaten ${ }^{15}$ einen geeigneten, invarianten Kraftkonstantensatz übernehmen.

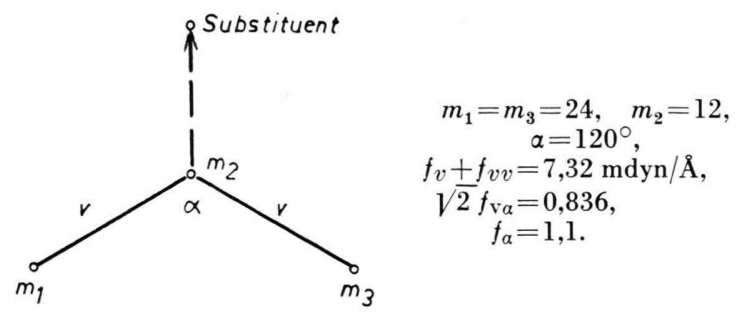

Abb. 1. Ersatzmodell für eine Phenylgruppe.

Die Beschreibung der Valenzschwingungen durch Kraftkonstanten ist wegen der Nichtdiagonalglieder in den $F$-Matrizen in einem ausgedehnten Lösungsbereich möglich. Bei der Eingrenzung dieser Lösungsmannigfaltigkeit gingen wir nach folgenden Gesichtspunkten vor:

1. Untersuchung der Lösungsbereiche der F-Matrizen bei Schwingungsproblemen vom Rang $n=2$.

2. Annahme nicht zu großer Wechselwirkungskonstanten, die im allgemeinen etwa um eine Zehnerpotenz kleiner sind als die Valenzkraftkonstanten.

3. Bei großem Energieunterschied zweier gekoppelter Schwingungen $\left(G_{11} F_{11} \sim \lambda_{1} \gg G_{22} F_{22} \sim \lambda_{2}\right)$ soll zur Potentialenergie einer Schwingung die zweite Kraftkonstante möglichst wenig beitragen. Der Anteil der Wechselwirkungskonstanten an der Potentialenergie soll von ähnlich kleiner Größenordnung und entgegengesetztem Vorzeichen sein.

4. Annahme von Erwartungsbereichen für Valenzkraft- und Wechselwirkungskonstanten durch Übertragung von Kraftkonstanten in einer Reihe ähnlicher Substanzen.

5. Berücksichtigung der Frequenzverschiebung bei der Substitution von ${ }^{16} \mathrm{O}$ durch ${ }^{18} \mathrm{O}$, die auf unterschiedliche Kopplung stark anspricht.

10 D. N. Kursanov, M. E. Volpin u. Yu. D. Koreshiov, J. Allg. Chem. (russ.) (engl. Übers.) 30, 2855 [1960].

11 F. Toda u. K. AKagi, Tetrahedron Letters 34, 3735 [1968].

12 E. Osawa, K. Kitamura u. Z. I. Yoshida, J. Am. Chem. Soc. 89, 3814 [1967].

13 J.Agranat u. E. D. Bergmann, Tetrahedron Letters 1966 , 2373.

14 H. J. Becher u. K. Ballein, Z. Phys. Chem. Frankfurt 54, 302 [1967].

15 H. J. BECHER U. F. Höfler, in Vorbereitung. 


\section{Cyclopropenylium-Verbindungen}

Die in Tab. 1 angeführten Spektren von $\mathrm{C}_{3} \mathrm{Cl}_{3}{ }^{+}$ und $\mathrm{C}_{3} \mathrm{Br}_{3}{ }^{+}$sind einer Arbeit von West ${ }^{16}$ entnommen, in der auch Kraftkonstanten berechnet werden.

\begin{tabular}{lrcrr}
\hline \multicolumn{2}{c}{$\begin{array}{c}\text { Klasse } \mathrm{A}^{\prime}{ }_{1} \\
\mathrm{C}_{3} \mathrm{Cl}_{3}^{+}\end{array}$} & \multicolumn{2}{c}{$\mathrm{C}_{3} \mathrm{Cl}_{3}^{+}$} & $\mathrm{C}_{3} \mathrm{Br}_{3}^{+}$ \\
\hline$v_{\mathrm{S}} \mathrm{Ring}$ & 1791 & $v_{\text {as }} \mathrm{Ring}$ & 1320 & 1276 \\
$v_{\mathrm{S}} \mathrm{CX}$ & 459 & $v_{\text {as }} \mathrm{CX}$ & 740 & 575 \\
& & $\delta \mathrm{CX}$ & 200 & \\
\hline
\end{tabular}

Tab. 1. Schwingungsfrequenzen von Trihalogencyclopropenylium-Kationen in $\mathrm{cm}^{-1}\left(\mathrm{~A}_{1}^{\prime}\right.$-Schwingungen von $\mathrm{C}_{3} \mathrm{Br}_{3}{ }^{+}$wurden nicht gemessen).

Nach WEST ergab das Urey-Bradley-Feld für die C - C-Valenzkraftkonstante $(6,31-6,74 \mathrm{mdyn} / \AA)$ deutlich höhere Wert als in fünf- und sechsgliedrigen aromatischen Ringen $(5,39-5,59 \mathrm{mdyn} / \AA)$; die $\mathrm{C}-\mathrm{Cl}$ Valenzkraftkonstante variierte jedoch stark $(2,99$ bis $5,08 \mathrm{mdyn} / \AA)$, je nachem ob die Wechselwirkungskonstante zweier $\mathrm{C}-\mathrm{Cl}$-Bindungen mit 0 oder einem kleinen positiven Wert $(0,08)$ eingesetzt wurde. Ebenso waren die Ergebnisse nach einem einfachen Valenzkraftmodell erwartungsgemäß unbefriedigend.

Für unsere Betrachtungen verwenden wir die aus Abb. 2 ersichtlichen inneren Koordinaten und die nebenstehenden Symmetriekoordinaten.

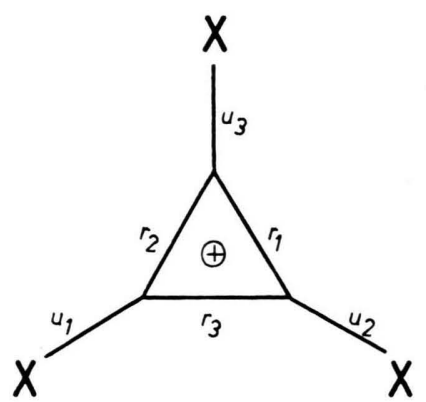

Abb. 2. Innere Koordination, Symmetriekoordinaten und F-Matrizen von $\mathrm{C}_{3} \mathrm{X}_{3}^{+}$.

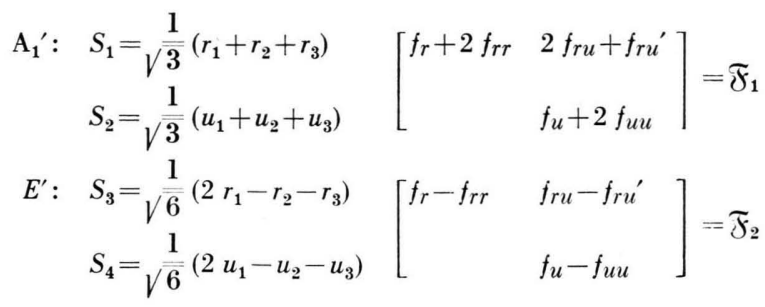

Da nach Weglassung der tiefen Deformationsschwingung in $E^{\prime}\left(200 \mathrm{~cm}^{-1}\right)$ jeweils nur zwei Schwingungen

16 R. West, A. Sado u. S. W. Tobey, J. Am. Chem. Soc. 88, 2488 [1966]. in einer Symmetrieklasse liegen, lassen sich die Abhängigkeiten von $F_{11}\left(F_{33}\right)$ und $F_{22}\left(F_{44}\right)$ vom Nebendiagonalglied $F_{12}\left(F_{34}\right)$ leicht überblicken.

Für die Auswahl der unten angeführten Lösungsbereiche aus den gesamten Lösungsellipsen, deren graphische Darstellung hier weggelassen wurde, waren folgende Kriterien bestimmend:

a) Der Potentialenergieanteil $V_{i}\left(F_{i i}\right)$ der Ringkoordinate $S_{i}(i=1,3)$ an der Ringschwingung soll nahe bei 1 liegen;

b) die CCl-Kraftkonstanten $\left(f_{u}\right)$ in den beiden Rassen sollen von möglichst ähnlicher Größe sein, $f_{u u}$ sollte also möglichst klein sein;

c) in den Wechselwirkungsgliedern sollte der $f_{r u}{ }^{\prime}$-Anteil möglichst klein sein;

d) ein sinnvolles Verhältnis zwischen den Kraftkonstanten von $\mathrm{C}_{3} \mathrm{Cl}_{3}{ }^{+}$und $\mathrm{C}_{3} \mathrm{Br}_{3}{ }^{+}$soll erreicht werden.

Danach ergeben sich folgende Wertebereiche für die Symmetriekraftkonstanten (in mdyn $/ \AA$ ) :

$$
\begin{array}{cc}
\mathrm{C}_{3} \mathrm{Cl}_{3}{ }^{+}\left(\mathrm{A}_{1}{ }^{\prime}\right): & \mathrm{C}_{3} \mathrm{Cl}_{3}{ }^{+}\left(\mathrm{E}^{\prime}\right): \\
f_{r}+2 f_{r r}=8,1-8,4 & f_{r}-f_{r r}=7,30-7,65 \\
2 f_{r u}+f_{r u}=1,8-2,0 & f_{r u}-f_{r u}^{\prime}=1,15-1,30 \\
f_{u}+2 f_{u u}=4,42-4,45 & f_{u}-f_{u u}=5,10-5,25 \\
\operatorname{mit} \quad V_{1}\left(F_{11}\right)=1,08-1,11 & \text { mit } \quad V_{3}\left(F_{33}\right)=0,86-0,91 \\
& \mathrm{C}_{3} \mathrm{Br}_{3}{ }^{+}\left(\mathrm{E}^{\prime}\right): \\
f_{r}-f_{r r}=7,4-7,7 \\
f_{r u}-f_{r u}^{\prime}=0,95-1,15 \\
f_{u}-f_{u u}=3,8-3,9 \\
\text { mit } V_{3}\left(F_{33}\right)=0,94-0,95
\end{array}
$$

Die aus den Mittelwerten dieser Linearkombination erhaltenen Kraftkonstanten des $\mathrm{C}_{3} \mathrm{Cl}_{3}{ }^{+}$sind in Tab. 2 zusammengestellt.

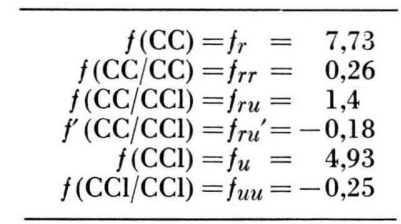

Tab. 2. Kraftkonstanten von $\mathrm{C}_{3} \mathrm{Cl}_{3}{ }^{+}$in mdyn/ $\mathrm{A}$.

Die Bindungs-Bindungs-Wechselwirkungskonstante im Ring $\left(f_{r r}\right)$ berechnen wir als klein und positiv. Die Wechselwirkungskonstante zwischen einer CClund einer gegenüberliegenden CC-Bindung $\left(f_{r u}{ }^{\prime}\right)$ kann nach unserem Modell nicht vernachlässigt werden; würde man sie mit 0 einsetzen, so käme man zu einem Kraftkonstantensatz mit zu großen $f_{r^{-}}$und $f_{r u}$-Werten und einer unbefriedigenden Potentialenergieverteilung. Ebenso muß der CCl-CCl-Wechselwirkungskonstante $\left(f_{u u}\right)$ eine kleine negative Größe zugeordnet werden.

Die CC-Kraftkonstante des Dreirings ist nach unseren Rechnungen größer als die CC-Kraftkonstante im Benzol, die nach least-square-Rech- 
nungen von SchereR ${ }^{17}$ und Califano ${ }^{18} 6,73$ bzw. $6,43 \mathrm{mdyn} / \AA$ beträgt. Überraschend hoch ist der Wert für $f(\mathrm{CCl})=4,93 \mathrm{mdyn} / \AA$ und auch für $f(\mathrm{CBr})-f(\mathrm{CBr} / \mathrm{CBr})=3,8-3,9 \mathrm{mdyn} / \AA$, verglichen mit $f(\mathrm{CX})$ in Chlorbenzol $(3,77 \mathrm{mdyn} / \AA)$ oder Brombenzol $(2,89 \mathrm{mdyn} / \AA)$. Wie aus den angegebenen Lösungsbereichen ersichtlich ist, sind die CXKraftkonstanten relativ unempfindlich gegenüber Änderungen im Wechselwirkungsglied $F_{12}\left(F_{34}\right)$. Unseren Rechnungen am TriphenylcyclopropenyliumKation legen wir ein koplanares Modell der Sym-

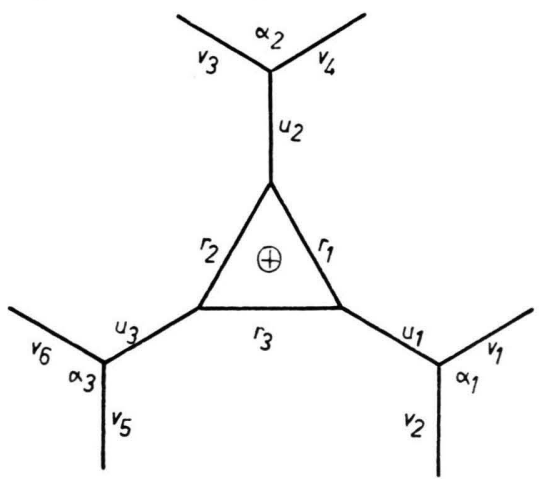

$$
\begin{aligned}
& \mathrm{A}_{1}{ }^{\prime}: \quad S_{1}=\frac{1}{\sqrt{3}}\left(r_{1}+r_{2}+r_{3}\right) \\
& E^{\prime}: \quad S_{5}=\frac{1}{\sqrt{2}}\left(r_{1}-r_{2}\right) \\
& S_{2}=\frac{1}{\sqrt{3}}\left(u_{1}+u_{2}+u_{3}\right) \\
& S_{6}=\frac{1}{\sqrt{2}}\left(u_{1}-u_{3}\right)
\end{aligned}
$$

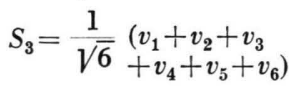

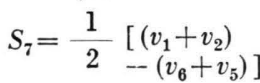

$$
\begin{aligned}
& S_{4}=\frac{1}{\sqrt{3}}\left(\alpha_{1}+\alpha_{2}+\alpha_{3}\right) \\
& S_{8}=\frac{1}{\sqrt{2}}\left(a_{1}-a_{3}\right)
\end{aligned}
$$

Abb. 3. Innere Koordinaten und Symmetriekoordinaten von $\mathrm{C}_{3} \mathrm{ph}_{3}{ }^{+}$. metrie $D_{3 h}$ zugrunde, in dem die Phenylgruppen durch gewinkelte Dreimassengruppen repräsentiert werden (Abb. 1, Abb. 3).

Mit einer vom $\mathrm{C}_{3} \mathrm{Cl}_{3}{ }^{+}$übertragenen CC-Kraftkonstante im Dreiring $\left(f_{r}\right)$ und dem übernommenen Kraftkonstantensatz des Phenylmodells und Erwartungsbereichen der übrigen Kraftkonstanten ergab sich bereits eine gute Übereinstimmung zwischen berechneten und beobachteten Frequenzen ${ }^{19,} 20$ in den Klassen $\mathrm{A}_{1}{ }^{\prime}$ und $\mathrm{E}^{\prime}$. Eine Frequenzanpassung nach dem Eigenvektorübertragungsverfahren ${ }^{21}$ erbrachte sodann die in Tab. 3 angeführten Ergebnisse. Die Spalte PEV enthält die Hauptdiagonalglieder der Matrizen der Potentialenergieverteilung.

Die gefundenen hohen CC-Kraftkonstanten im und am Cyclopropenyliumring (7,74 bzw. 5,86 mdyn $/ \AA$ ) werden durch Messungen der Bindungsabstände gestützt. SundaralingaM ${ }^{22}$ gibt für $\mathrm{C}_{3} \mathrm{ph}_{3}{ }^{+}\left(\mathrm{ph}=\mathrm{C}_{6} \mathrm{H}_{5}\right)$ einen CC-Abstand im Dreiring von $1,373 \AA$ an, der etwas kürzer als der CC-Abstand im Benzol $(1,397 \AA)$ ist; der Wert des Abstandes C(Dreiring) - C (Sechsring) ist mit 1,436 deutlich kleiner als der üblicherweise zwischen zwei $\mathrm{sp}^{2}$-hybridisierten C-Atomen gefundene Abstand von $1,48 \AA$ (z. B. Diphenyl, Butadien). Die Phenylringe sind im Mittel um $13^{\circ}$ aus der Dreiecksebene herausgedreht. Berechnungen der Potentialenergieverteilung $V_{n}\left(F_{i i}\right)$ (Tab. 3) zeigen, daß die höchste

\begin{tabular}{|c|c|c|c|c|c|c|c|c|c|}
\hline $\begin{array}{l}F_{11}=8,25 \\
F_{22}=5,69 \\
F_{33}=F_{77}=7,32 \\
F_{44}=F_{88}=1,1\end{array}$ & & \multicolumn{4}{|c|}{$\begin{array}{l}F_{12}=1,875 \\
F_{23}=F_{67}=1,164 \\
F_{24}=F_{68}=-0,232 \\
F_{13}=F_{14}=F_{57}=F_{58}=0\end{array}$} & \multicolumn{4}{|c|}{$\begin{array}{l}F_{34}=F_{78}=0,836 \\
F_{55}=7,48 \\
F_{66}=5,95 \\
F_{56}=1,125\end{array}$} \\
\hline \multicolumn{2}{|l|}{ Koordinate } & \multicolumn{2}{|c|}{$\mathrm{PEV}\left[V_{n}\left(F_{i i}\right)\right]$} & \multirow{2}{*}{\multicolumn{2}{|c|}{$\begin{array}{c}\text { Koordinate } \\
S_{i}\left(\mathrm{E}^{\prime}\right)\end{array}$}} & \multirow[b]{2}{*}{$n=1$} & \multicolumn{2}{|c|}{$\operatorname{PEV}\left[\tilde{V}_{n}\left(F_{i i}\right)\right.$} & \multirow[b]{2}{*}{4} \\
\hline$S_{i}\left(\mathrm{~A}^{\prime}\right)$ & $n=1$ & 2 & 3 & & & & 2 & 3 & \\
\hline$S_{1}$ & 1,02 & 0,01 & 0,01 & 0,03 & $S_{5}$ & 0,55 & 0,34 & 0,08 & 0,05 \\
\hline$S_{2}$ & 0,27 & 0,50 & 0,08 & 0,30 & $S_{6}$ & 0,68 & 0,20 & 0,09 & 0,11 \\
\hline$S_{3}$ & 0,00 & 0,66 & 0,43 & 0,06 & $S_{7}$ & 0,06 & 0,45 & 0,61 & 0,01 \\
\hline$S_{4}$ & 0,00 & 0,37 & 0,23 & 0,52 & $S_{8}$ & 0,05 & 0,24 & 0,03 & 0,79 \\
\hline Frequenzen ber. & 1844 & 1117 & 685 & 300 & & 1409 & 1119 & 735 & 484 \\
\hline beob. & 1845 & 1117 & 685 & - & & 1411 & 1117 & - & - \\
\hline
\end{tabular}
Schwingung in $\mathrm{A}_{1}^{\prime}\left(1845 \mathrm{~cm}^{-1}\right)$ überwiegend durch die Dreiringpulsation $\left(S_{1}\right)$ bestimmt ist, während die übrigen Schwingungen erwartungsgemäß sehr stark gekoppelt erscheinen. An der Frequenz 1117

Tab. 3. F-Matrix (mdyn/ $\AA$ ) des $\mathrm{C}_{3} \mathrm{ph}_{3}{ }^{+}$in $\mathrm{A}_{1}{ }^{\prime}$ und $\mathrm{E}^{\prime}$, Vergleich von beobachteten und berechneten Frequenzen (cm ${ }^{-1}$ ) und Potentialenergieverteilung.

17 J. R. SCHERER, Spectrochim. Acta 20, 345 [1964].

18 N. Neto, M. Scrocco u. S. Califano, Spectrochim. Acta 22, 1981 [1966].

19 B. Schrader u. W. Meier, Z. Naturforsch. 21 a, 480 [1966].

20 J. Chatt u. R. GuY, Chem. Ind. 1963, 212; DMS-Karten AO 571, AO 533, Verlag Chemie, Weinheim 1965.
21 H. J. Becher u. R. Mattes, Spectochim. Acta 23 A, 2449 [1967].

22 M. Sundaralingam u. L. H. Jensen, J. Am. Chem. Soc. 88, 198 [1966]. 
$\mathrm{cm}^{-1}$ sind beispielsweise die Pulsations- $\left(S_{3}\right)$ und Deformationsschwingung $\left(S_{4}\right)$ des Phenylrings und die exocyclische CC-Valenzschwingung $\left(S_{2}\right)$ beteiligt.

\section{Tetrahalogencyclopropene}

Die höchste symmetrische Gerüstschwingung von Cyclopropenderivaten, die hauptsächlich durch $v(\mathrm{C}=\mathrm{C})$ charakterisiert sein sollte, liegt meist bei höheren Wellenzahlen als die $v(\mathrm{C}=\mathrm{C})$ bei offenkettigen Olefinen. Nach den Daten der Tab. 4 unterliegt sie einer starken Kopplungsbeeinflussung insbesondere durch die an den Olefin-C-Atomen befindlichen Substituenten ${ }^{6,27}$.

\begin{tabular}{lllcr}
\hline $\mathrm{R}$ & $\mathrm{R}^{\prime}$ & $\mathrm{R}^{\prime \prime}$ & $v\left(\mathrm{~cm}^{-1}\right)$ & Lit. \\
\hline $\mathrm{H}$ & $\mathrm{H}$ & $\mathrm{H}$ & 1641 & 23 \\
$\mathrm{H}$ & $\mathrm{CH}_{3}$ & $\mathrm{H}$ & 1780 & 6 \\
$\mathrm{H}$ & $\mathrm{CH}_{3}$ & $\mathrm{CH}$ & 1885 & 6 \\
$\mathrm{CH}_{3}$ & $\mathrm{H}$ & $\mathrm{H}$ & 1632 & 2 \\
$\mathrm{CH}_{3}$ & $\mathrm{H}$ & $\mathrm{D}$ & 1578 & 6 \\
$\mathrm{CH}_{3}$ & $\mathrm{D}$ & $\mathrm{D}$ & 1525 & 6 \\
$\mathrm{CH}_{3}$ & $\mathrm{H}$ & $\mathrm{CH}_{3}$ & 1768 & 2 \\
$\mathrm{CH}_{3}$ & $\mathrm{D}$ & $\mathrm{CH}_{3}$ & 1718 & 6 \\
$\mathrm{CH}_{3}$ & $\mathrm{CH}$ & $\mathrm{CH}_{3}$ & 1877 & 6 \\
$\mathrm{H}$ & $\mathrm{C}_{6} \mathrm{H}_{5}$ & $\mathrm{C}_{6} \mathrm{H}_{5}$ & 1826 & 24 \\
$\mathrm{~F}$ & $\mathrm{Cl}$ & $\mathrm{Cl}$ & 1760 & 25 \\
$\mathrm{~F}$ & $\mathrm{CF}$ & $\mathrm{CF}_{3}$ & 1820 & 25 \\
$\mathrm{Cl}$ & $\mathrm{Cl}$ & $\mathrm{Cl}$ & 1811 & 26 \\
$\mathrm{Br}$ & $\mathrm{Br}$ & $\mathrm{Br}$ & 1764 & 26 \\
\hline
\end{tabular}

Tab. 4. Frequenzen mit überwiegendem $\nu \mathrm{C}=\mathrm{C}$-Charakter in substituierten Cyclopropenen

$$
\mathrm{R}^{\prime} \overbrace{\mathbf{R}^{\prime \prime}}^{\mathbf{R}_{2}}
$$

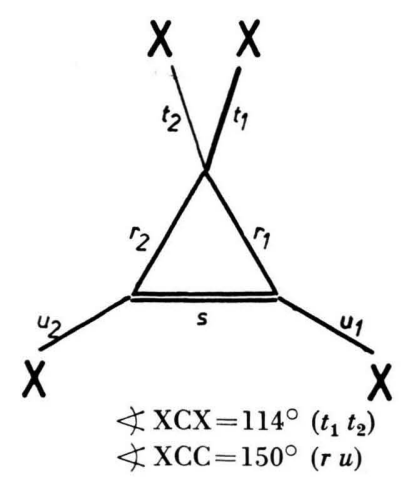

Vollständige und zugeordnete Schwingungsspektren existieren nur von $\mathrm{C}_{3} \mathrm{Cl}_{4}$ und $\mathrm{C}_{3} \mathrm{Br}_{4}{ }^{26}$. Sie sind für die betrachteten Symmetrieklassen $A_{1}$ und $B_{2}$ (Symmetrie $\mathrm{C}_{2 \mathrm{v}}$ ) unter Weglassung der bei niedrigen Wellenzahlen gelegenen Deformationsschwingungen in Tab. 5 zusammengestellt, und zwar ist wiederum jene Schwingungsbewegung angegeben, die für die jeweilige Frequenz am stärksten bestimmend ist.

\begin{tabular}{lrrcrr}
\hline \multicolumn{1}{c}{$\mathrm{A}_{1}$} & $\mathrm{C}_{3} \mathrm{Cl}_{4}$ & $\mathrm{C}_{3} \mathrm{Br}_{4}$ & $\mathrm{~B}_{2}$ & $\mathrm{C}_{3} \mathrm{Cl}_{4}$ & $\mathrm{C}_{3} \mathrm{Br}_{4}$ \\
\hline$v_{\mathrm{s}} \mathrm{CX}_{2}$ & 411 & 244 & & & \\
$\nu_{\mathrm{s}} \mathrm{CC}_{2}$ & 1148 & 1121 & $v_{\mathrm{as}} \mathrm{CC}_{2}$ & 1057 & 1000 \\
$\nu \mathrm{C}=\mathrm{C}$ & 1811 & 1764 & $\nu_{\mathrm{as}} \mathrm{CX}$ & 689 & 582 \\
$\nu_{\mathrm{s}} \mathrm{CX}$ & 616 & 491 & & & \\
\hline
\end{tabular}

Tab. 5. Schwingungsfrequenzen von $\mathrm{C}_{3} \mathrm{Cl}_{4}$ und $\mathrm{C}_{3} \mathrm{Br}_{4}$ in $\mathrm{cm}^{-1}$.

Abbildung 4 enthält die Symmetriekoordinaten von $\mathrm{C}_{3} \mathrm{X}_{4}$ in $\mathrm{A}_{1}$ und $\mathrm{B}_{2}$ sowie die $F$-Matrix von $\mathrm{B}_{2}$.

Die graphische Darstellung des Lösebereiches der $F$-Matrix in der Klasse $\mathrm{B}_{2}$ des $\mathrm{C}_{3} \mathrm{Cl}_{4}$ zeigte uns, daß ein Nebendiagonalglied $F_{56}$ von $1,32 \mathrm{mdyn} / \AA$ den unteren Grenzwert für reelle Lösungen darstellt. Wesentlich größere Werte der Wechselwirkungskraftkonstanten sind in Anlehnung an die Ergebnisse bei $\mathrm{C}_{3} \mathrm{X}_{3}{ }^{+}$unwahrscheinlich und führen auch auf sehr ungünstige Potentialenergieverteilungen.

Unter der Voraussetzung, daß in der höheren Frequenz der Anteil der CC-Valenzbewegung überwiegt, folgt aus der Lösungsellipse:

$$
\begin{aligned}
& F_{55}=f_{r}-f_{r r}=4,5-4,7, \\
& F_{56}=f_{r u}-f_{r u}{ }^{\prime}=1,32-1,33 \text { und } \\
& F_{66}=f_{u}-f_{u u}=5,0-4,80 \mathrm{mdyn} / \AA .
\end{aligned}
$$

Abb. 4. Innere Koordinaten und Symmetriekoordinaten von $\mathrm{C}_{3} \mathrm{X}_{4}$ sowie $F$-Matrix der Klasse $\mathrm{B}_{2}$.

${ }^{23}$ K. B. Wiberg u. B. J. Nist, J. Am. Chem. Soc. 83, 1226 [1961].

24 A. KREBS u. B. Schrader, unveröffentlicht.

${ }_{25}$ S. V. Tobey u. R. West, J. Am. Chem. Soc. 88, 2481 [1966].
26 M. Iтo, Spectrochim. Acta 22, 1581 [1966].

27 C. F. Wilcox JR. u. R. R. Craig, J. Am. Chem. Soc. 83, 3866 [1961]. 
Im $\mathrm{C}_{3} \mathrm{Br}_{4}$, bei dem die Kopplung weniger stark ist, ergibt sich im gleichen Bereich von $f_{r}-f_{r r}$

$$
f_{r u}-f_{r u}{ }^{\prime}=1,21-1,30
$$

und $\quad f_{u}-f_{u u}=4,2-4,06 \mathrm{mdyn} / \AA$.

Die C-Halogenkraftkonstanten sind somit von ähnlicher Größenordnung wie in den $\mathrm{C}_{3} \mathrm{X}_{3}{ }^{+}$-Ionen.

Bei den Rechnungen in der Klasse $A_{1}$ gehen wir von Erwartungswerten der Valenzkraftkonstanten aus. Die die CC-Einfachbindung $\left(S_{2}\right)$ betreffende Symmetriekraftkonstante $f(\mathrm{CC})+f(\mathrm{CC} / \mathrm{CC})$ sollte 4,6-4,9 mdyn/ $\AA$ betragen. Als Anhaltspunkt für die Kraftkonstante der $\mathrm{C}=\mathrm{C}$-Doppelbindung diente der von BECHER ${ }^{28}$ für das Äthylen angegebene Wert von $9,15 \mathrm{mdyn} / \AA$ (in ${ }^{28}$ wird ausführlich auf die Problematik bei den Normalkoordinatenanalysen des Äthylens eingegangen). Wegen des verkürzten Bindungsabstandes ( $1,30 \AA$ in Cyclopropen gegenüber

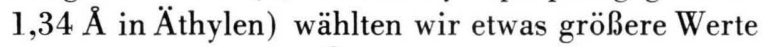
von $9,8-10,0 \mathrm{mdyn} / \AA$.

Die Größen der einzelnen Nebendiagonalkraftkonstanten wurden in Anlehnung an die vorher ermittelten Bereiche gewählt. Nach einer Anpassung einer geeigneten Ausgangs- $F$-Matrix an die beobachteten Frequenzen erhielten wir verfeinerte Kraftkonstanten, die in Tab. 6 zusammen mit den berechneten Frequenzwerten und den Hauptdiagonalgliedern der Potentialenergieverteilungsmatrizen aufgeführt sind.

Die Summe von $f(\mathrm{CCl})+f(\mathrm{CCl} / \mathrm{CCl})$ der $\mathrm{CCl}_{2}$ Gruppe mußte mit $4,3 \mathrm{mdyn} / \AA$ relativ hoch eingesetzt werden; dieser Wert kann mit einem etwas größeren Fehler behaftet sein, da die Frequenz $v_{\mathrm{s}} \mathrm{CCl}_{2}$ bei $411 \mathrm{~cm}^{-1}$ bereits im Bereich der nicht berücksichtigten Ringdeformationsschwingungen liegt und außerdem eine Unsicherheit im CICCl-Winkel (hier zu $114^{\circ}$ angenommen) sich auf das zugehörige $G$-Matrixelement auswirkt. Nach der Potentialenergieverteilung sind alle inneren Koordinaten in den betrachteten Frequenzen zum Teil beträchtlich gekoppelt.

Tabelle 7 enthält Mittelwerte der Ring- und $\mathrm{C}-\mathrm{Cl}$-Kraftkonstanten des $\mathrm{C}_{3} \mathrm{Cl}_{4}$. Mit entsprechend abgeänderten C-Halogenkraftkonstanten und geringfügig veränderten Wechselwirkungsgliedern lassen sich auch die $\mathrm{A}_{1}$-Frequenzen des $\mathrm{C}_{3} \mathrm{Br}_{4}$ in guter Näherung wiedergeben.

\begin{tabular}{lllll}
\hline$f(\mathrm{C}-\mathrm{C})$ & $=f_{r}=4,60$ & $f(=\mathrm{C}-\mathrm{Cl})$ & $=f_{u}=4,75$ \\
$f(\mathrm{CC} / \mathrm{CC})=f_{r r}=0,05$ & $f(=\mathrm{CCl}=\mathrm{CCl})=f_{u u}=-0,15$ \\
$f(\mathrm{C}=\mathrm{C})=f_{s}=9,90$ & $f(\mathrm{C}-\mathrm{C} / \mathrm{CCl})$ & $=f_{r u}=1,04$ \\
$f(\mathrm{C}=\mathrm{C} / \mathrm{CC})=f_{r s}=0,29$ & $f^{\prime}(\mathrm{C}-\mathrm{C} / \mathrm{CCl})$ & $=f_{r u}=-0,29$ \\
& & $f(\mathrm{C}=\mathrm{C} / \mathrm{CCl})$ & $=f_{s u}=0,69$
\end{tabular}

Tab. 7. Kraftkonstanten von $\mathrm{C}_{3} \mathrm{Cl}_{4}$ in mdyn/ $\AA$.

\section{Cyclopropenone}

Eine kurze Charakterisierung der beiden typischen Cyclopropenonbanden bei $1830-1865$ und 1590 bis $1660 \mathrm{~cm}^{-1}$ wurde bereits in der Einleitung dieser Arbeit gegeben, eine ausführliche Zusammenstellung der bisherigen spektroskopischen Untersuchungsergebnisse an Cyclopropenonen findet sich bei Krebs und Schrader ${ }^{1}$. Am Beispiel des Diphenylcyclopropenons wurde deutlich, daß insbesondere die ${ }^{18} \mathrm{O}$-Markierung wertvolle Zusatzinformationen zum Verständnis der Schwingungsspektren liefern kann. Aus diesem Grunde untersuchten wir nun auch ${ }^{18} \mathrm{O}$-substituiertes Dimethylcyclopropenon.

\section{a) Dimethylcyclopropenon}

Die IR- und Raman-Spektren von Dimethylcyclopropenon sind in Abb. 5 gezeigt und in Tab. 8 zusammengefaßt. Die ${ }^{18} \mathrm{O}$-substituierte Verbindung lag dabei in 60-proz. Anreicherung vor; die in der Spalte $\Delta v$ angegebenen Isotopenverschiebungen sind für vollständige ${ }^{18} \mathrm{O}$-Substitution extrapolierte Werte.

\begin{tabular}{|c|c|c|c|c|c|c|}
\hline & & & & & & \\
\hline & & Koordinate & & $\mathrm{PE}$ & $\left.\left.F_{i i}\right)\right]$ & \\
\hline & & $S_{i}$ & $n=1$ & 2 & 3 & 4 \\
\hline$F_{11}=4,30$ & $\begin{array}{l}F_{12}=0,90 \\
F_{13}=F_{14}=0\end{array}$ & $S_{1}$ & 0,55 & 0,22 & 0,00 & 0,27 \\
\hline$F_{22}=4,71$ & $F_{23}=0,41$ & $S_{2}$ & 0,04 & 0,84 & 0,16 & 0,03 \\
\hline$F_{33}=9,90$ & $F_{24}=0,75$ & $S_{3}$ & 0,00 & 0,15 & 0,78 & 0,09 \\
\hline$F_{44}=4,60$ & $F_{34}=0,97$ & $S_{4}$ & 0,30 & 0,00 & 0,18 & 0,56 \\
\hline & & Frequenzen & 414 & 1151 & 1812 & 616 \\
\hline
\end{tabular}

Tab. 6. $F$-Matrix in mdyn $/ \AA$, berechnete Frequenzen in $\mathrm{cm}^{-1}$ und Potentialenergieverteilung in der $\operatorname{Rasse} \mathrm{A}_{1}$ des $\mathrm{C}_{3} \mathrm{Cl}_{4}$.

28 H. J. BeCher, Fortschr. Chem. Forsch. 10, 156 [1968]. 


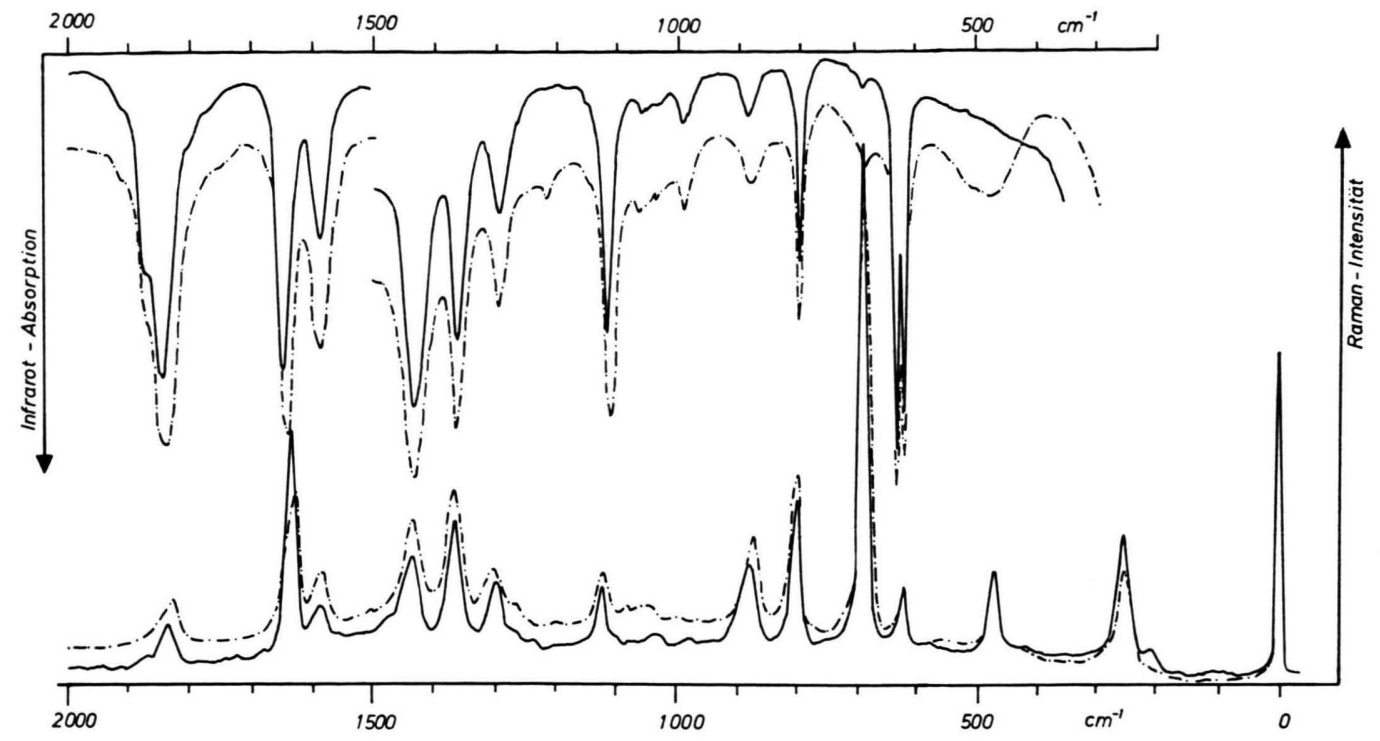

Abb. 5. Infrarot- (obere Kurven) und Raman-Spektren (un tere Kurven) von Dimethylcyclopropenon. —— ${ }^{16} \mathrm{O}-\mathrm{Verbin}$ dung; $-\cdot-\cdot-\cdot-$ mit $60 \%{ }^{18} \mathrm{O}$ markierte Verbindung.

\begin{tabular}{|c|c|c|c|c|c|}
\hline \multicolumn{2}{|c|}{160} & \multicolumn{2}{|c|}{160} & \multirow[b]{2}{*}{$\Delta v$} & \multirow[b]{2}{*}{ Zuordnung* } \\
\hline $\mathrm{Ra}$ & IR & $\mathrm{Ra}$ & IR & & \\
\hline $258(8) d p$ & $\begin{array}{l}\text { nicht } \\
\text { gem. }\end{array}$ & 256 & $\begin{array}{l}\text { nicht } \\
\text { gem. }\end{array}$ & 3 & Def. \\
\hline $472(2) \mathrm{p} ?$ & - & 472 & - & - & \\
\hline $621(1) \mathrm{dp}$ & $619(5)$ & 620 & 617 & 3 & $\delta \mathrm{CO}$ \\
\hline- & $632(6)$ & - & 630 & 3 & $\gamma \mathrm{CO}$ \\
\hline $688(10) p$ & $688(0)$ & 686 & - & 3 & $v_{\mathrm{s}} \mathrm{C}-\mathrm{C}\left(\mathrm{H}_{3}\right), \mathrm{A}_{1}$ \\
\hline $802(3) \mathrm{dp}$ & $800(4)$ & 802 & 799 & 1 & $\begin{array}{l}v_{\text {as }} \mathrm{C}-\mathrm{C}\left(\mathrm{H}_{3}\right) \\
\text { und } v_{\mathrm{as}} \mathrm{CC}_{2}, \mathrm{~B}_{2}\end{array}$ \\
\hline $888(2) \mathrm{p} ?$ & $888(0)$ & 881 & - & 11 & $v_{\mathrm{S}} \mathrm{CC}_{2}, \mathrm{~A}_{1}$ \\
\hline $1121(1) \mathrm{p} ?$ & $1119(5)$ & 1115 & 1113 & 10 & $\begin{array}{l}v_{\text {as }} \mathrm{C}-\mathrm{C}\left(\mathrm{H}_{3}\right) \\
\text { und } v_{\mathrm{as}} \mathrm{CC}_{2}, \mathrm{~B}_{2}\end{array}$ \\
\hline $1302(1) d p$ & $1296(3)$ & 1305 & 1299 & - & \\
\hline $1369(3) \mathrm{p} ?$ & $1365(4)$ & 1369 & 1364 & - & $\delta_{\mathrm{s}} \mathrm{CH}_{3}$ \\
\hline $1437(2) \mathrm{dp}$ & $1432(5)$ & 1437 & 1435 & - & $\delta_{\mathrm{as}} \mathrm{CH}_{3}$ \\
\hline $1590(0)$ & $1587(5)$ & 1587 & 1585 & 5 & \\
\hline $1644(4) p$ & $1647(10)$ & 1637 & 1637 & 12 & $\int v \mathrm{C}=\mathrm{C}, \mathrm{A}_{1}$ \\
\hline $1841(1) \mathrm{p}$ & $1843(10)$ & 1831 & 1835 & 13 & $v \mathrm{C}=\mathrm{O}, \mathrm{A}_{1}$ \\
\hline
\end{tabular}

* Die angegebene innere Koordinate ist am stärksten an der betreffenden Schwingung beteiligt.

Tab. 8. IR- und Raman-Spektren von Dimethylcyclopropenon.

Die Zuordnung der Gerüstvalenzschwingungen der Klasse $\mathrm{A}_{1}$ folgt klar aus Intensitäten und Polarisationszuständen der Raman-Linien. Für die beiden $\mathrm{B}_{2}$-Schwingungen erscheinen die Banden bei 1121 und $802 \mathrm{~cm}^{-1}$ am wahrscheinlichsten; die hohe Isotopenverschiebung der ersteren kann allerdings nicht erklärt werden. Von den Methyl-Deformationsschwingungen sind $\delta_{\mathrm{s}} \mathrm{CH}_{3}$ und $\delta_{\mathrm{as}} \mathrm{CH}_{3}$ gut, $\varrho \mathrm{CH}_{3}$ hingegen nicht zu erkennen (1300 $\mathrm{cm}^{-1}$ erscheint dafür zu hoch).

Für die Kraftkonstantenrechnungen werden im Strukturbild Ia die Methylgruppen als Punktmassen $\left(m_{\mathrm{R}}=15\right)$ betrachtet und zu $\mathrm{C}_{3} \mathrm{X}_{4}$ analoge innere
Koordinaten verwendet. Die Symmetriekoordinate für $v \mathrm{C}=\mathrm{O}$ wird $S_{1}=t$; die übrigen Symmetriekoordinaten und auch die Bedeutung der $F$-Matrix der Klasse $B_{2}$ entsprechen den Ausdrücken bei $\mathrm{C}_{3} \mathrm{X}_{4}$ (Abb. 4).

Aus der Untersuchung des Lösebereiches der Kraftkonstanten in der Klasse $\mathrm{B}_{2}$, die nur 2 Valenzschwingungen enthält, geht hervor, daß man auch bei Cyclopropenonsystemen mit relativ großen Wechselwirkungskraftkonstanten zwischen Dreiring- und Substituentenbindungen zu rechnen hat: Reelle Lösungen für $F_{55}$ und $F_{66}$ ergeben sich erst bei $F_{56} \geqq 1,14$ mdyn/ $/$. Ähnlich wie bei $\mathrm{C}_{3} \mathrm{Cl}_{4}$ erhält man sinnvolle Kraftkonstantenwerte und Potentialenergieverteilungen nur im $\mathrm{Be}$ reich maximaler Frequenzkopplung bei

$$
F_{56}=f_{r u}-f_{r u^{\prime}}=1,14-1,15 \mathrm{mdyn} / \AA .
$$

$F_{55}=f_{r}-f_{T r}$ und $F_{66}=f_{u}-f_{u u}$ betragen hier 4,97 bis 5,25 bzw. $4,35-4,15$ mdyn/ $\AA$. Die vereinfachte Berücksichtigung der Methylgruppen, die Unsicherheit über den RCC-Winkel und die Vernachlässigung der Deformationsschwingungen führen hier und auch bei den $\mathrm{A}_{1}$-Rechnungen (vgl. Tab. 9) zu zu niedrigen und daher nur begrenzt signifikanten Kraftkonstanten der exocyclischen CC-Bindung $\left(f_{u}\right)$. Nach den vorhergehenden Ergebnissen und aus dem Vergleich mit Toluol sollte man sie zu etwa 4,8 mdyn/ $/$ erwarten.

In der Klasse $A_{1}$ des Dimethylcyclopropenons (Tab. 9) können durch die zusätzlichen Isotopendaten nicht alle 8 berücksichtigten Kraftkonstanten der $F$-Matrix exakt ermittelt werden, da von den je 4 gemessenen Frequenzen der ${ }^{16} \mathrm{O}$ - und der ${ }^{18} \mathrm{O}$-Verbindung eine durch die Produktregel fixiert wird. 


\begin{tabular}{|c|c|c|c|c|c|c|c|c|c|c|}
\hline \multirow{8}{*}{$\begin{array}{l}F_{11}=10,95 \\
F_{22}=5,0 \\
F_{33}=9,2 \\
F_{44}=4,1\end{array}$} & \multirow{8}{*}{$\begin{array}{l}F_{12}=0,75 \\
F_{13}=F_{14}=0 \\
F_{23}=0,1 \\
F_{24}=0,60 \\
F_{34}=1,05\end{array}$} & \multirow{2}{*}{$\begin{array}{c}\text { Koordinate } \\
S_{i}\end{array}$} & \multicolumn{3}{|c|}{$\operatorname{PEV}\left[V_{n}\left(F_{i i}\right)\right]$} & \multirow[b]{2}{*}{4} & \multicolumn{4}{|c|}{ Eigenvektoren $\left(L_{i, n}\right)$} \\
\hline & & & $n=1$ & 2 & 3 & & $n=1$ & 2 & 3 & 4 \\
\hline & & $S_{1}$ & 0,52 & 0,15 & 0,30 & 0,05 & 0,31 & 0,08 & 0,21 & 0,03 \\
\hline & & $S_{2}$ & 0,38 & 0,55 & 0,00 & 0,10 & $-0,39$ & 0,23 & $-0,01$ & 0,07 \\
\hline & & $S_{3}$ & 0,20 & 0,15 & 0,67 & 0,01 & $-0,21$ & $-0,09$ & 0,34 & 0,02 \\
\hline & & $S_{4}$ & 0,06 & 0,12 & 0,15 & 0,72 & 0,17 & $-0,11$ & $-0,24$ & 0,22 \\
\hline & & Frequenzen & 1845 & 887 & 1647 & 692 & 1845 & 887 & 1647 & 692 \\
\hline & & $\Delta v$ & 14,0 & 15,5 & 12,1 & 6,0 & & & & \\
\hline
\end{tabular}

Tab. 9. $F$-Matrix in mdyn/ $\AA$, berechnete Frequenzen und Isotopenverschiebungen in $\mathrm{cm}^{-1}$, Potentialenergieverteilung und Eigenvektoren in der Rasse $A_{1}$ des Dimethylcyclopropenons.

Orientierende Frequenzrechnungen ergaben, da $\beta$ bereits kleine Änderungen in jeder der berücksichtigten Kraftkonstanten die Verteilung des Isotopeneffektes auf die einzelnen Schwingungen empfindlich beeinflussen. Daher ermöglicht die Isotopensubstitution in Verbindung mit den bisher gewonnenen Größenordnungen der Wechselwirkungsglieder gute Bereichseingrenzungen für die Valenz- und Wechselwirkungskraftkonstanten bw. Aussagen über die Relationen der einzelnen Kraftkonstanten zueinander.

Bei einer Resonanzstabilisierung durch die zwitterionische Cyclopropenyliumform Ib sollte die Bindungsordnung $\operatorname{der} \mathrm{C}=\mathrm{O}$-Bindung herabgesetzt sein und zum anderen ein Bindungsausgleich im Dreiring zu einer niedrigeren $\mathrm{C}=\mathrm{C}$ - und einer höheren $\mathrm{C}-\mathrm{C}$ Kraftkonstanten als in Cyclopropenen führen. Die in Tab. 9 aufgeführten Ergebnisse einer Frequenzrechnung zeigen, daß dies für die $\mathrm{C}=\mathrm{C}$ - und die $\mathrm{C}$ - C-Kraftkonstante verglichen mit $\mathrm{C}_{3} \mathrm{Cl}_{4}$ tatsächlich zutrifft. Leider liegen für die $\mathrm{C}=\mathrm{O}$-Kraftkonstante in der Literatur noch keine ausreichend gesicherten Werte vor ${ }^{28-30}$. Am zuverlässigsten erscheint noch die $\mathrm{C}=\mathrm{O}$-Kraftkonstante im Formaldehyd, die nach etwas unterschiedlichen Berechnungen von Mecke und Mitarb. ${ }^{29}$ sowie Cleveland ${ }^{30}$ bei $(12,7 \pm 0,6) \mathrm{mdyn} / \AA$ liegt. Gegenüber diesem Wert ist die von uns ermittelte Kraftkonstante im Dimethylcyclopropenon deutlich erniedrigt.

Die Wiedergabe der Isotopenverschiebungen durch die $F$-Matrix der Tab. 9 ist in Hinblick auf die durch die Extrapolation vergrößerte Fehlerbreite der Messungen und der getroffenen Vereinfachungen in der Potentialfunktion befriedigend. Die komplizierten Kopplungsbeeinflussungen kommen auch in der Potentialenergieverteilung zum Ausdruck. Die $\mathrm{C}=\mathrm{O}$ Valenzkoordinate ist an drei Systemschwingungen

29 L. Beckmann, L. Gutjahr u. R. Mecke, Spectrochim. Acta 21, 307 [1965]. beteiligt. Zur höchsten Frequenz liefert außer $\nu \mathrm{C}=\mathrm{O}$ auch $v_{\mathrm{s}} \mathrm{CC}_{2}$ einen sehr großen Energieanteil. Eine weiter gehende Beschreibung der Schwingungsformen liefert die Eigenvektormatrix $(L)$. Die der $\mathrm{C}=\mathrm{O}$ - und $\mathrm{C}=\mathrm{C}$-Koordinate entsprechenden Elemente besitzen in den Frequenzen bei 1845 und $1647 \mathrm{~cm}^{-1}$ entgegengesetztes bzw. gleiches Vorzeichen (Tab. 9). Die in ${ }^{1}$ und ${ }^{4}$ vorgeschlagene Beschreibung dieser beiden Banden als ungleichphasige und gleichphasige gekoppelte Schwingung $\operatorname{der} \mathrm{C}=\mathrm{O}$. und $\mathrm{C}=\mathrm{C}$-Koordinate findet sich somit durch die Rechnung bestätigt. Die Kopplung $\operatorname{der} \nu \mathrm{C}=\mathrm{O}$ und $v \mathrm{C}=\mathrm{C}$ erfolgt über die CC-Einfachbindung, deren Eigenvektoren bei den beiden Schwingungen höherer Frequenz das entgegengesetzte Vorzeichen wie die der $\mathrm{C}=$ O-Gruppe, bei der Schwingung bei $887 \mathrm{~cm}^{-1}$ das gleiche Vorzeichen besitzen.

Durch Verkleinerung der $(\mathrm{C}=\mathrm{O})$ - und gleichzeitige Vergrößerung der $(\mathrm{C}=\mathrm{C})$-Kraftkonstante kommen wir zu etwa gleich großen Isotopenverschiebungen bei den beiden höchsten Frequenzen. Wählt man sehr große Bindungs-Bindungs-Wechselwirkung im Ring, so erhält die Frequenz um $1840 \mathrm{~cm}^{-1}$ überwiegenden $\mathrm{C}=\mathrm{C}$-Charakter, der Isotopeneffekt wird stärker auf die Frequenz bei $1645 \mathrm{~cm}^{-1}$ abgewälzt. Die Wahl sehr kleiner Wechselwirkungskonstanten von Ring- und Substituentenbindungen führt zu einem „Keton-Modell“", bei dem die Bande bei $1840 \mathrm{~cm}^{-1}$ hauptsächlich durch $\nu \mathrm{C}=\mathrm{O}$ bestimmt wird und den entsprechenden Isotopeneffekt zeigt, während jene bei $1645 \mathrm{~cm}^{-1}$ nahezu unverändert bleibt. In allen Fällen erfährt die Bande um $880 \mathrm{~cm}^{-1}$ die größte Isotopenverschiebung.

\section{b) Diphenylcyclopropenon}

In Diphenylcyclopropenon wurde die ${ }^{16} \mathrm{O}-{ }^{18} \mathrm{O}$. Isotopenverschiebung nur an den beiden typischen Cyclopropenonbanden in Abhängigkeit von Lösungsmitteleffekten untersucht ${ }^{1}$. Einige Meßdaten sind in

30 M. G. Pillai Krishna u. F. F. Cleveland, J. Mol. Spectry. 6, 465 [1961]. 
Tab. 10 noch einmal zusammengestellt; in Fällen, wo die Banden infolge Fermi-Resonanz aufgespalten waren, wird der Mittelwert der beiden Frequenzen benutzt. Die Raman-Linien bei 1120 (m), 870 (ss) und $690 \mathrm{~cm}^{-1}$ werden ebenfalls $A_{1}$-Frequenzen zugeordnet.

Wie bei der Behandlung des Triphenylcyclopropenylium-Systems ersetzen wir die Phenylringe durch koplanare gewinkelte Dreimassengruppen mit gleichbleibendem Kraftkonstantensatz. Die Symmetriekoordinaten der Klasse $A_{1}$ entsprechen dann denen beim Dimethylderivat $\left(S_{1}-S_{4}\right)$, vermehrt um die beiden Koordinaten $\left(S_{5}, S_{6}\right)$ des Phenylmodells (Abb. 1).

\begin{tabular}{cllllll}
\hline $\begin{array}{c}\text { Lösungs- } \\
\text { mittel }\end{array}$ & $\begin{array}{l}\text { Cyclo- } \\
\text { hexan }\end{array}$ & $\Delta v$ & $\mathrm{CCl}_{4}$ & $\Delta v$ & $\mathrm{CHCl}_{3}$ & $\Delta v$ \\
\hline $16 \mathrm{O}$ & 1861 & & 1853 & & 1854,5 & \\
$18 \mathrm{O}$ & 1843,5 & & 1838 & & 1840 & \\
$16 \mathrm{O}$ & 1643 & & 1637 & & 1625 & \\
$18 \mathrm{O}$ & 1633 & & 1626 & & 1615 & \\
\hline
\end{tabular}

Tab. 10. Lage der beiden typischen Cyclopropenonbanden von ${ }^{16} \mathrm{O}$ - und ${ }^{18} \mathrm{O}$-Diphenylcyclopropenon in verschiedenen Lösungsmitteln sowie Isotopenverschiebungen in $\mathrm{cm}^{-1}$.

Die Größen der Kraftkonstanten (Tab. 11), ihr Einfluß auf die Isotopenverschiebungen, die PEV sowie die Eigenvektoren (in Tab. 11 nur teilweise aufgeführt) entsprechen im wesentlichen den Verhältnissen im Dimethylcyclopropenon. Der erniedrigte Wert der $\mathrm{C}=\mathrm{C}$-Doppelbindungskraftkonstante könnte auf einen etwas stärkeren Bindungsausgleich im Diphenylderivat zurückgehen. Die Kraftkonstante der exocyclischen CC-Bindung ist deutlich geringer als in $\mathrm{C}_{3} \mathrm{ph}_{3}{ }^{+}$. Wie bei diesem zeigt die PEV eine starke Kopplung zwischen der Phenyl-Ringschwingung und der exocyclischen CC-Valenzschwingung.

Valenzkraftkonstanten und Mittelwerte der Wechselwirkungskraftkonstanten von Cyclopropenonen finden sich in Tab. 12.

\section{Zusammenfassung}

Die hier beschriebenen Normalkoordinatenanalysen an Cyclopropenderivaten gestatten eine befriedigende Wiedergabe der beobachteten Frequenzen und der Isotopenverschiebungen bei ${ }^{18} \mathrm{O}$-Substitution der Cyclopropenone. Wegen der beträchtlichen Mischungen zwischen den inneren Koordinaten in den einzelnen Normalschwingungen können die Schwingungsformen durch die Potentialenergieverteilung angemessener wiedergegeben werden als durch eine Betrachtung charakteristischer Gruppenfrequenzen.

Die gute Úbertragbarkeit vergleichbarer Valenzkraftkonstanten und auch der Größenordnung der

$\begin{array}{ll}F_{11}=10,8 & F_{12}=0,67 \\ F_{22}=5,25 & F_{23}=0,22 \\ F_{33}=8,7 & F_{24}=0,9 \\ F_{44}=5,2 & F_{34}=1,15 \\ F_{55}=7,32 & F_{45}=0,85 \\ F_{66}=1,1 & F_{46}=-0,24 \\ & F_{56}=0,836\end{array}$

$F_{13}=F_{14}=F_{15}=F_{16}=0$

$F_{25}=F_{26}=F_{35}=F_{36}=0$

$\begin{array}{ll}\text { Eigenvektoren: } & L_{1,1}=0,31 \\ & L_{1,3}=0,20\end{array}$

\begin{tabular}{ccccccc}
\hline $\begin{array}{c}\text { Koordinate } \\
S_{i}\end{array}$ & $n=1$ & 2 & $\mathbf{3}$ & $\mathbf{3}$ & 5 & 6 \\
\hline$S_{1}$ & 0,54 & 0,19 & 0,25 & 0,01 & 0,01 & 0,01 \\
$S_{2}$ & 0,40 & 0,62 & 0,00 & 0,00 & 0,01 & 0,01 \\
$S_{3}$ & 0,16 & 0,13 & 0,64 & 0,04 & 0,01 & 0,03 \\
$S_{4}$ & 0,06 & 0,02 & 0,24 & 0,41 & 0,10 & 0,28 \\
$S_{5}$ & 0,00 & 0,01 & 0,01 & 0,65 & 0,41 & 0,06 \\
$S_{6}$ & 0,00 & 0,00 & 0,01 & 0,35 & 0,23 & 0,54 \\
\hline Frequenzen & 1853 & 873 & 1638 & 1122 & 681 & 355 \\
$\Delta v$ & 14,6 & 19,3 & 10,3 & 0,6 & 1,1 & 2,5 \\
\hline
\end{tabular}

$$
\begin{aligned}
& L_{3,1}=-0,20 \\
& L_{3,3}=0,34
\end{aligned}
$$

Tab. 11. F-Matrix in mdyn/ $\AA$, berechnete Frequenzen und Is otopenverschiebungen in $\mathrm{cm}^{-1}$, Potentialenergieverteilung und Eigenvektoren in der Rasse $A_{1}$ des Diphenylcyclopropenons.

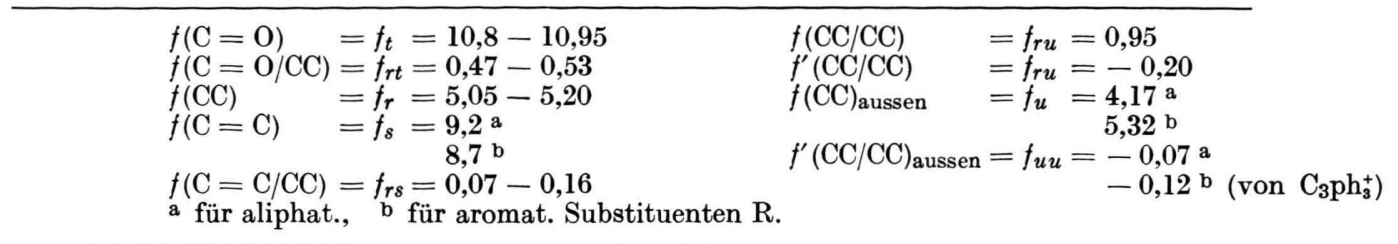

Tab. 12. Kraftkonstanten von Cyclopropenonen in mdyn/A. 
berücksichtigten Wechselwirkungskraftkonstanten innerhalb der betrachteten Verbindungen berechtigt zu dem Schluß, daß diese Kraftkonstanten die Bindungsverhältnisse sinnvoll beschreiben. Sie zeigen, $\mathrm{da} ß$ die Beteiligung der Betain-Struktur bei den Cyclopropenonen in den Kraftkonstanten zum Ausdruck kommt, obwohl die Abweichungen gegenüber einer „normalen “ $\mathrm{C}=\mathrm{O}$ - und $\mathrm{C}=\mathrm{C}$-Doppelbindungskraftkonstante geringer sind als man vielleicht erwartet hätte. Ihre beiden Schwingungen bei 1840 und $1620 \mathrm{~cm}^{-1}$ können nicht eindeutig als $\nu \mathrm{C}=0$. oder $v \mathrm{C}=\mathrm{C}$ zugeordnet werden, vielmehr ist die potentielle Energie dieser Schwingungen über alle Bindungen des Ringsystems verteilt. Angenähert kann man aber von einer Gegentakt- (1840) und einer Gleichtakt-Schwingung $\left(1620 \mathrm{~cm}^{-1}\right) \operatorname{der} \mathrm{C}=\mathrm{O}$ - und der $\mathrm{C}=\mathrm{C}$-Bindung gleichzeitig sprechen. Dabei ist aber die Schwingung bei $1840 \mathrm{~cm}^{-1}$ stärker als auf die $\mathrm{C}=\mathrm{O}$-Bindung, die bei $1620 \mathrm{~cm}^{-1}$ stärker auf die $\mathrm{C}=\mathrm{C}$-Bindung lokalisiert. Dies ist im Wider- spruch zu der Zuordnung dieser Schwingungen auf Grund der Frequenzverschiebung mit der Polarität des Lösungsmittels.

$$
\begin{aligned}
& \text { Darstellung des }{ }^{18} \text { O-Dimethylcyclo- } \\
& \text { propenons }
\end{aligned}
$$

$738 \mathrm{mg} \quad(9 \mathrm{mMol})$ Dimethylcyclopropenon wurden mit $339 \mathrm{mg}(17 \mathrm{mMol}) \mathrm{H}_{2}{ }^{18} \mathrm{O}\left(90,735 \%{ }^{18} \mathrm{O} ; 0,295 \%\right.$ $\left.{ }^{17} \mathrm{O}\right)$ vermischt, das durch Einleiten von gasförmigem $\mathrm{HCl}$ auf $\mathrm{pH} 1$ gebracht worden war. Das Reaktionsgemisch blieb bei $25{ }^{\circ} \mathrm{C}$ unter Lichtausschluß $64 \mathrm{Std}$. stehen und wurde bei 30 Torr destilliert; dabei gingen bei etwa $30^{\circ} 220 \mathrm{mg}$ Wasser über. Der Rückstand lieferte bei $56-58^{\circ} / 0,8$ Torr $665 \mathrm{mg}$ (90\%) Dimethylcyclopropenon mit $55 \%{ }^{18} \mathrm{O}$. Nach einem weiteren analogen Austausch enthielt das Dimethylcyclopropenon $60 \%{ }^{18} \mathrm{O}$ (massenspektrometrisch bestimmt) .

Die Autoren sind Herrn Prof. Dr. H. J. Becher (Universität Münster/W.) für viele wertvolle Anregungen zu Dank verpflichtet. Einer von uns (F. H.) dankt der Deutschen Forschungsgemeinschaft für ein Stipendium. - Die Rechnungen wurden am Recheninstitut der Universität Münster durchgeführt. 